\title{
KIMURA DISEASE: A CASE REPORT
}

K. Venkatramireddy ${ }^{1}$, Ramakrishna Reddy², James Premsagar $\mathrm{M}^{3}$, Shanker Reddy ${ }^{4}$, P. Karthik Prakash ${ }^{5}$

\section{HOW TO CITE THIS ARTICLE:}

K. Venkatramireddy, Ramakrishna Reddy, James Premsagar M, Shanker Reddy, P. Karthik Prakash. "Kimura Disease: A Case Report". Journal of Evolution of Medical and Dental Sciences 2015; Vol. 4, Issue 40, May 18; Page: 7083-7088, DOI: $10.14260 /$ jemds/2015/1030

ABSTRACT: Kimura's disease is a rare chronic inflammatory disorder of unknown etiology, primarily seen in young Asian males.1,2 The disease is characterized by a triad of painless subcutaneous masses in head and neck region, blood and tissue eosinophilia and moderately elevated serum immunoglobulin E levels. It is also characterized by angiolymphoid proliferation with infiltration mainly by eosinophils, some plasma cells, lymphocytes, and mast cells and a predilection for the head and neck region. There is associated involvement of the major salivary gland and multiple regional lymph nodes. Although it was first described in the Chinese literature in 1937 under the designation of "eosinophilic hyperplastic lymphogranuloma," it was not until 1948 that the disease to become widely known as KD when Kimura and Ishikawa reported it in the Japanese literature. ${ }^{3}$ Here we report a case of Kimura's disease with clinico-pathological differential diagnosis.

KEYWORDS: Kimura, Head Neck Masses, Subcutaneous Masses, Eosinophilia.

CASE REPORT: A 50-years-old man presented with symptoms of generalised weakness and pruritis and swelling. Multiple cervical, axillary, and inguinal lymphadenopathies were found on physical examination. Blood studies revealed peripheral eosinophilia (15\%); raised absolute eosinophil count (4800 cells/cumm); mildly elevated WBC count (15000 cells/cumm) and an elevated ESR (150 mm/1st hr).

Patient was then subjected to a ultarsonographic examination. Sonography showed a conglomerate of multiple enlarged lymph nodes in the bilateral cervical, axillary, inguinal region and porta hepatis with a homogeneous internal echo pattern and near normal maintenance of nodal hilar region showing increased flow on colour Doppler (RI $=0.53$; PI=0.76 ). We also found few well defined tiny rounded hypoechoic subcutaneous nodules in the left suboccipital region. The sizes of cervical nodes varied between 5- $10 \mathrm{~mm}$.

Figure 1: Ultarsonography showing a well-defined rounded hypoechoic subcutaneous nodule in the suboccipital region.

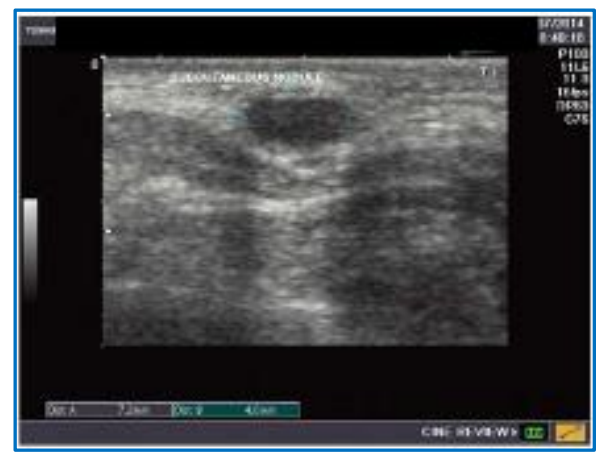

\section{Fig. 1}




\section{CASE REPORT}

Figure 2: USG shows multiple well defined hypoechoic lymph nodal masses within the b/l submandibular glands.

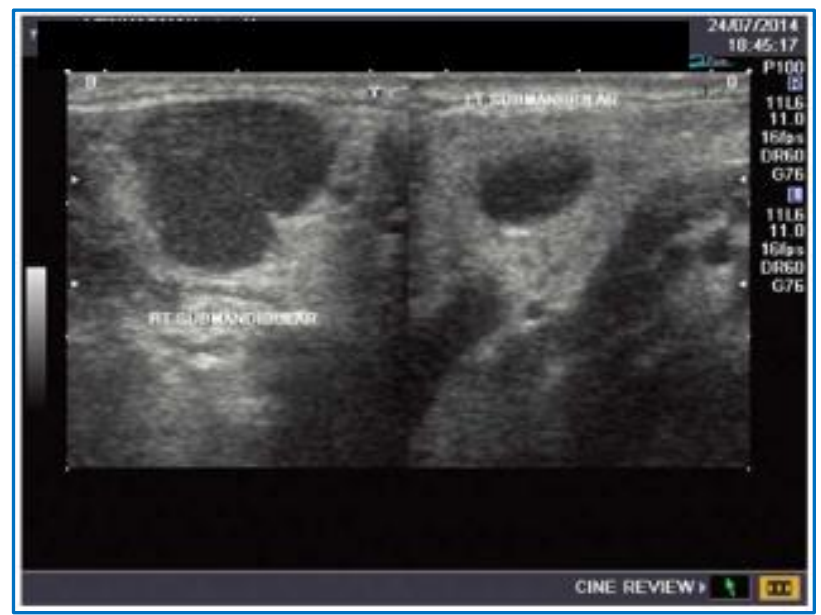

\section{Fig. 2}

For further evaluation the patient was subjected to contrast enhanced CT study.

Contrast-enhanced CT showed multiple and extensive areas of conglomerated lymphadenopathy involving the entire abdomen-encasing of the celiac axis along with bilateral cervical, axillary and inguinal lymphadenopathy. The lesions showed variable degree of enhancement.

FNAC from the lymphnode revealed inconclusive granulomatous changes. In order to confirm the diagnosis lymphnodal biopsy was performed which revealed distorted architecture of the lymphnode which is replaced by a mixture of inflammatory cells predominantly eosinophils,

Moderate number of macrophages, along with hyalinization of few blood vessels.

All the above mentioned features favour the diagnosis of kimura disease.

Figure 3: USG abdomen shows e/o multiple enlarged hypoechoic para aortic and iliac nodes.

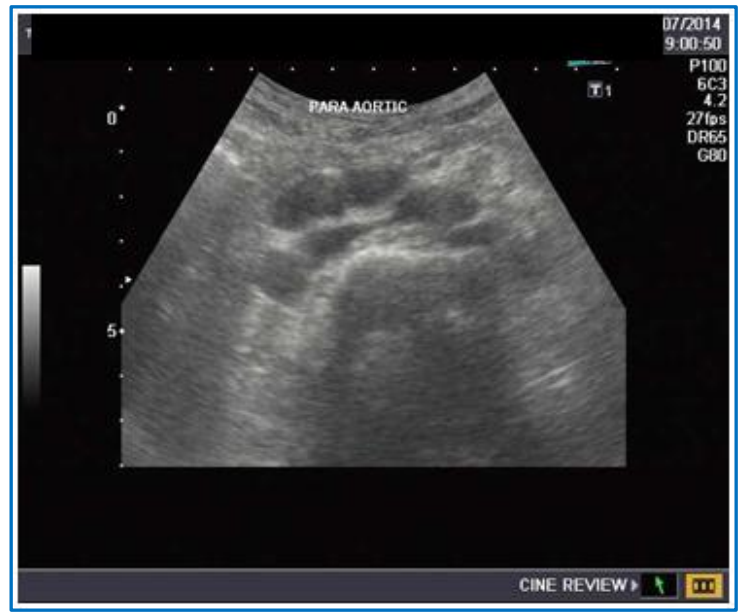

Fig. 3 


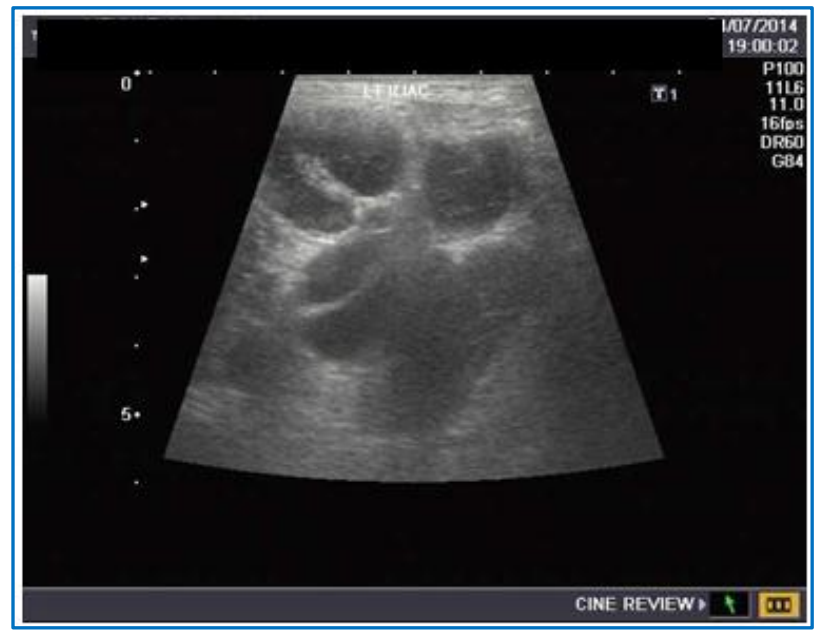

Fig. 4

DISCUSSION: Kimura's disease first described in1937 in Chinese literature by H. T. Kimm and C. Szeto and they termed it as "eosinophilic hyperplastic lymphogranuloma". The disease became widely known as Kimura's disease after Kimura and colleagues reported two cases of unusual granulation combined with hyperplastic changes of lymphoid tissue. KD is characterized histologically by proliferation of folliculoid structures, which are infiltrated by eosinophils, plasma cells, lymphocytes, and mast cells and demonstrate associated vascular proliferation and stromal fibrosis. Fibrosis can be present in the early stage of the lesions and may be replaced later by hyalinization. ${ }^{4,5} \mathrm{~A}$ definitive diagnosis can only made by the lymph nodal biopsy findings.

The etiopathogenesis of KD remains unknown and it is considered nowadays as an allergic disease and it seems to be a systemic immunological disorder. Eosinophilia and increased serum IgE levels make KD be considered a CD4 (+) T helper $2(\mathrm{Th} 2)$ allergic reactions. Th2 cells would produce interleukins (IL) IL-4, IL-5 and IL-13, which, in turn, would act on B cells favoring the production of antigen-specific IgE. Th2 cell proliferation and the overexpression of cytokines would play an essential role in the development of the disease. ${ }^{6}$

It has been speculated that a viral or parasitic trigger may alter T-cell immunoregulation or induce an IgE-mediated type 1 hypersensitivity, resulting in the release of eosinophilotrophic cytokines. ${ }^{5,7}$

Clinically, KD primarily affects those of Asian descent in their second, third, and fourth decades of life with a male predilection, with a 3.5:1 to 9:1 male-to-female ratio. Most patients present with firm, painless, single, or multiple subcutaneous lesions in the head and neck, especially in the parotid and submandibular regions. Associated lymphadenopathy is frequent, with a reported incidence of $42 \%-100 \%$.

The variability in the degree of enhancement on CT and MR images and the signal intensity on MR images are thought to be attributed to the different degrees of fibrosis and vascular proliferation contained in the individual lesions. Abundant vascular proliferation may explain the presence of enhancement and signal-intensity-void structures on MR images. 


\section{CASE REPORT}

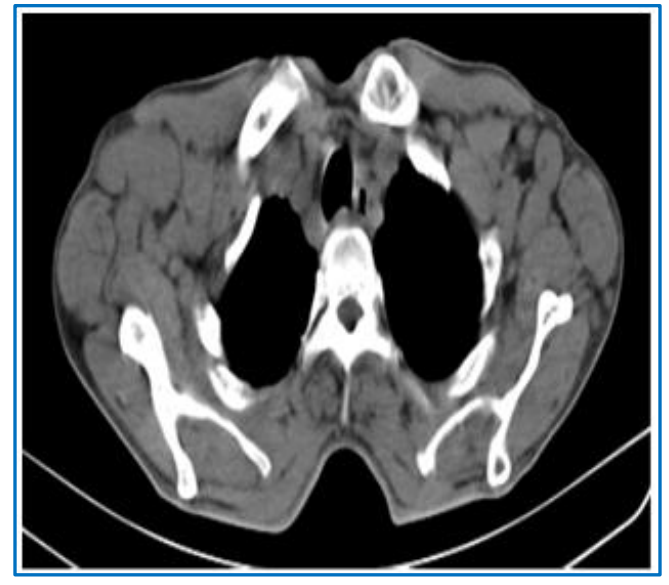

Fig. 5

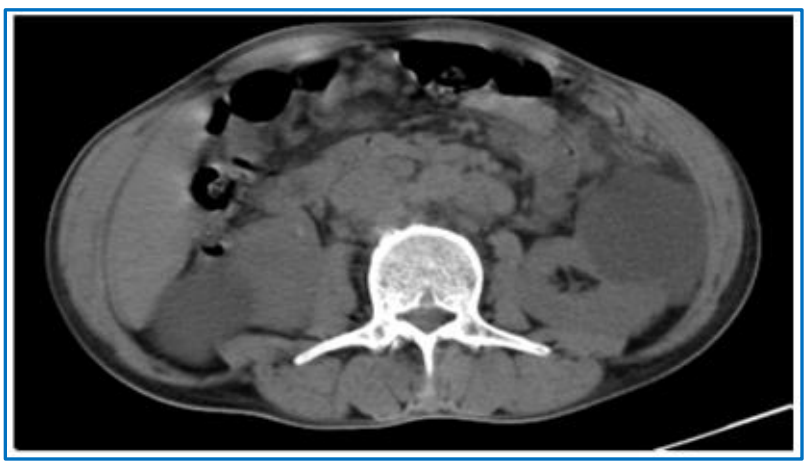

Fig. 7

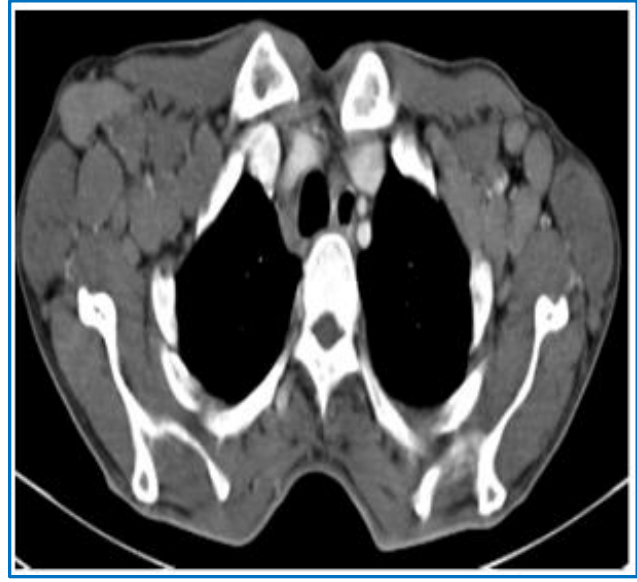

Fig. 6

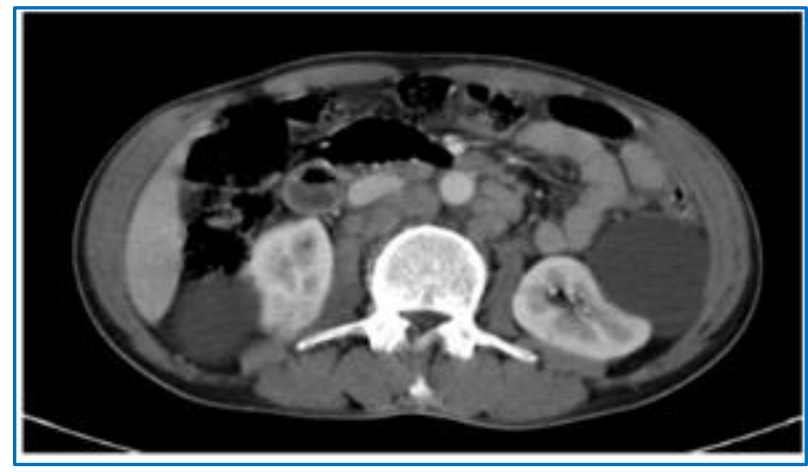

Fig. 8

Pre and post contrast CT sections of chest and abdomen with e/o multiple well defined round to oval shaped variably enhancing soft tissue density lesions (lymphnodes) in the b/l axillary, paracaval and para aortic regions.

Although lymphoma and metastatic lymphadenopathy may have similar presentations, the irregular margination of the lesions and the distribution of the lesions, as well as the long clinical course as seen in KD, are unusual.

Enhanced lymphadenopathy associated with tuberculosis usually demonstrates central hypoattenuation and peripheral rim enhancement, whereas enlarged lymph nodes enhance homogeneously in KD. KDcan be confused clinically and histologically with angiolymphoid hyperplasia with eosinophilia (ALHE), also known as epitheloid hemangioma, which is a rare but distinctive vascular tumor typically occurring in women. ALHE, however, usually presents as small erythematous dermal nodules in the head and neck that itch and bleed easily. There is less common association with lymphadenopathy and peripheral eosinophilia. Histologically, fibrosis is lacking in ALHE, whereas it is prominent at all stages in KD. Other entities that may present similarly to KD include nonhodgkins lymphoma, tuberculosis, sarcoidosis, posttransplant lymphoproliferative disorder, pseudotumor, Kuttner tumor, Castleman disease, and Sjogren syndrome. 
Treatment plan should aim at preservation of vital structures associated with the lesions \& cosmetic rehabilitation, while preventing recurrences. Treatment options range from observation \& follow-up of mild \& symptomatic cases to conservative surgical approach, medication (Systemic corticosteroids, cytotoxic agents, cyclosporin, and pentoxifylline,) \& radiotherapy in symptomatic \& recurrent cases. If the lesion is primary, localized or present in young age then surgical approach is more preferable. According to previous literature, only surgical approach had high incidence of recurrence. If the lesion is recurrent with systemic involvement, application of medication like corticosteroid and immunosuppressive agents have been shown to decrease size of the lesion. Irradiation should be considered in patients resistant to the steroid or to prevent the patient from deleterious effect of long term use of steroid. However Till date no definite treatment for KD is well established. ${ }^{8}$

CONCLUSION: In conclusion this paper draws attention on such a rare chronic inflammatory disease which mimics neoplastic and other chronic inflammatory conditions. This disease should be considered in differential diagnosis of patients presenting with head \& neck mass and lymphadenopathy and investigated accordingly as this disease has good prognosis. Knowledge of signature features of Kimura's disease put the physicians in a better position to evaluate its clinical outcome and optimal treatment regimen.

\section{REFERENCES:}

1. Kuo TT, Shih LY, Chan HL. Kimura's disease: involvement of regional lymph nodes and distinction from angiolymphoid hyperplasia with eosinophilia. Am J Surg Pathol 1988; 12: 84354.

2. Chen H, Thomson LD, Aguilera NS, et al. Kimura disease: a clinopathologic study of 21 cases. Am J Surg Pathol 2004; 28: 505-13.

3. Kimura T, Yoshimura S, Ishikawa E. Unusual granulation combined with hyperplastic changes of lymphatic tissue [in Japanese]. Trans Soc Pathol Jpn 1948; 37: 179-80.

4. Kung IT, Gibson JB, Bannatyne PM. Kimura's disease: a clinicopathological study of 21 cases and its distinction from angiolymphoid hyperplasia with eosinophilia. Pathology 1984; 16: 39-44.

5. Oguz KK, Ozturk A, Cila A. Magnetic resonance imaging findings in Kimura's disease. Neuroradiology 2004; 46: 855-58.

6. Briggs PL. Kimura disease is not angiolymphoid hyperplasia with eosinophilia: clinical and pathological correlation with literature review and definition of diagnostic criteria. Anais Brasileiros de Dermatologia 2006; 81 (2): 167-73.

7. Shetty AK, Beaty MW, McGuirt WF, et al. Kimura's disease: a diagnostic challenge. Pediatrics 2002; 110: e39.

8. Pitak-Arnnop P, Bellefqih S, Chaine A, Dhanuthai K, Bertrand JC, Bertolus C. Head and neck lesions of Kimura's disease: Exclusion of human herpesvirus-8 and Epstein-Barr virus by in situ hybridisation and polymerase chain reaction. An immunohistochemical study. Journal of Cranio-Maxillofacial Surgery 2010; 38 (4): 266-70. 


\section{CASE REPORT}

\section{AUTHORS: \\ 1. K. Venkatramireddy \\ 2. Ramakrishna Reddy \\ 3. James Premsagar M. \\ 4. Shanker Reddy \\ 5. P. Karthik Prakash}

\section{PARTICULARS OF CONTRIBUTORS:}

1. Professor \& HOD, Department of RadioDiagnosis, SVS Medical College.

2. Professor, Department of RadioDiagnosis, SVS Medical College.

3. Assistant Professor, Department of RadioDiagnosis, SVS Medical College.

FINANCIAL OR OTHER COMPETING INTERESTS: None
4. Professor, Department of Radio-Diagnosis, SVS Medical College.

5. Resident, Department of Radio-Diagnosis, SVS Medical College.

\section{NAME ADDRESS EMAIL ID OF THE}

\section{CORRESPONDING AUTHOR:}

Dr. P. Karthik Prakash,

Resident, Department of Radiology,

Yenugonda, Mahabubnagar,

Telangana.

E-mail: karthikpatti@gmail.com

Date of Submission: 18/11/2014.

Date of Peer Review: 19/11/2014.

Date of Acceptance: 11/05/2015.

Date of Publishing: 18/05/2015. 